\title{
Nota
}

\section{CAPACIDADE DE SUPORTE DE CARGA DO SOLO EM SISTEMAS DE PRODUÇÃO DE LARANJA CONSERVACIONISTAS}

\author{
Jonez Fidalski ${ }^{(1) *}$, Karina Maria Vieira Cavalieri-Polizeli( ${ }^{(2)}$, Cássio Antonio Tormena ${ }^{(3)}$, \\ Guilherme Anghinoni ${ }^{(3)}$ e Pedro Antonio Martins Auler ${ }^{(4)}$
}

\footnotetext{
(1) Instituto Agronômico do Paraná, Área de Solos, Paranavaí, Paraná, Brasil.

(2) Universidade Federal do Paraná, Departamento de Solos e Engenharia Agrícola, Curitiba, Paraná, Brasil.

(3) Universidade Estadual de Maringá, Departamento de Agronomia, Programa de Pós-graduação em Agronomia, Maringá, Paraná, Brasil.

(4) Instituto Agronômico do Paraná, Área de Fitotecnia, Paranavaí, Paraná, Brasil.

* Autor correspondente.

E-mail: fidalski@iapar.br
}

\begin{abstract}
RESUMO
Sistemas conservacionistas em pomares de laranja podem aumentar a capacidade de suporte de carga do solo minimizando os seus riscos de compactação. $O$ objetivo deste trabalho foi avaliar a capacidade de suporte de carga por meio da pressão de preconsolidação e sua dependência a conteúdo de água, densidade e carbono orgânico de um Argissolo Vermelho distrófico latossólico, após 18 anos de implantação de plantas de cobertura permanentes nas entrelinhas de um pomar de laranja. O delineamento experimental foi de blocos ao acaso, com três repetições. Foram estudados três tratamentos de manejo por meio de roçadas nas entrelinhas do pomar, desde o plantio do pomar em 1993: gramínea Paspalum notatum, leguminosa Arachis pintoi e vegetação espontânea. Cento e oito amostras indeformadas de solo foram coletadas em 2011 sob o rodado e entrerrodado das máquinas nas estrelinhas do pomar com textura arenosa nas camadas de $0,00-0,10 ~ m\left(87 \mathbf{~ g ~}^{-1}\right.$ de argila) e 0,10-0,20 m (122 $\mathrm{g} \mathrm{kg}^{-1}$ de argila). Determinaram-se as pressões de preconsolidação das curvas de compressão do solo $(25,50,100,200,400,800$ e $1.600 \mathrm{kPa})$ em conteúdos de água sob três potenciais $(-80,-330 \mathrm{e}-1.000 \mathrm{hPa})$, a densidade do solo e o teor de carbono orgânico do solo. A pressão de preconsolidação não foi dependente do conteúdo de água, da densidade do solo e do teor de carbono orgânico do solo. A pressão de preconsolidação sob o rodado na camada de $\mathbf{0 , 0 0 - 0 , 1 0 ~ m ~}$ foi menor nos tratamentos leguminosa e vegetação espontânea. A manutenção permanente da gramínea manejada com roçadas nas entrelinhas do pomar de laranja proporcionou maior capacidade de suporte de carga do solo sob o rodado na camada superficial arenosa.
\end{abstract}

Palavras-chave: compactação do solo, densidade do solo, plantas de cobertura, pressão de preconsolidação, solo arenoso. 


\title{
ABSTRACT: SOIL LOAD-BEARING CAPACITY IN ORANGE GROVE CONSERVATION SYSTEMS
}

\begin{abstract}
Conservation tillage systems in orange groves can increase soil load-bearing capacity minimizing their risks of soil compaction. The objective of this study was to evaluate load-bearing capacity through preconsolidation pressure and its dependence on water content, bulk density, and organic carbon of a Typic Paleudult soil 18 years after setting up permanent plant cover between the rows of an orange grove. A randomized block experimental design was used, with three replications. Three groundcover treatments subjected to mowing between the rows of the grove, as of establishment of the orange grove in 1993, were studied: Paspalum notatum grass, the Arachis pintoi legume, and spontaneous vegetation.

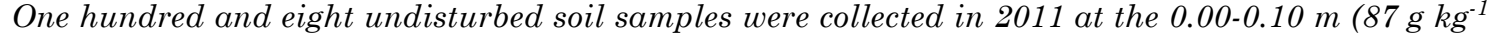
clay) and $0.10-0.20 \mathrm{~m}\left(122 \mathrm{~g} \mathrm{~kg}^{-1}\right.$ clay) depths under and between the wheel tracks of the machines between the rows of the orange grove consisting of sandy clay texture soil. Preconsolidation pressure was not dependent on soil water content, bulk density, or organic carbon content. Preconsolidation pressure from soil compression curves (25; 50; 100; 200; 400; 800; and 1,600 kPa) at water content under three potentials (-80; -330; and 1,000 hPa), soil bulk density, and soil organic carbon were determined. The preconsolidation pressure under the wheel tracks between the orange rows in the 0.00-0.10 m layer was lower in the legume and spontaneous vegetation treatments. Ongoing maintenance of the grass managed through mowing between the rows of the orange grove led to greater soil load-bearing capacity under the wheel tracks in the sandy surface layer.
\end{abstract}

Keywords: cover plants, preconsolidation pressure, soil bulk density, soil compaction, sandy soil.

\section{INTRODUÇÃO}

A citricultura paranaense caracteriza-se pela implantação de pomares de laranja adensados com espaçamentos inferiores a $7 \mathrm{~m}$ entre as linhas e a $4 \mathrm{~m}$ entre as plantas dentro das linhas. Nessa condição, o tráfego de máquinas se concentra próximo à projeção da copa das plantas dos pomares em produção, indicado pela deformação do solo da camada superficial com a formação de sulcos no local em que se concentra o tráfego do rodado das máquinas. A compactação do solo se deve ao intenso tráfego de aproximadamente 15 passadas anuais de máquinas e equipamentos nas entrelinhas dos pomares de laranja (Tersi e Rosa, 1995). Com o adensamento de plantas em pomares de laranja ocorre a aproximação das linhas de tráfego à projeção da copa das plantas, onde são realizadas as adubações.

A capacidade do solo de resistir às forças compressivas do tráfego de máquinas é de fundamental importância para a sustentabilidade dos sistemas de produção. A pressão de preconsolidação tem sido usada como indicador da capacidade de carga dos solos (Veenhof e McBride, 1996; Horn e Fleige, 2003; Imhoff et al., 2004; Rucknagel et al., 2007), uma vez que a aplicação de cargas maiores que essa pressão leva a uma compactação adicional para uma condição específica de manejo de solo (Dias Junior, 2000). A pressão de preconsolidação também tem sido avaliada em diferentes sistemas de manejo com plantas de cobertura do solo e tráfego de máquinas (Debiasi et al., 2008; Araujo-Junior, 2011; Kamimura et al., 2012). Em um pomar comercial de laranja variedade Pera com manejo das entrelinhas com roçada e aplicação de herbicida, Lima et al. (2004) verificaram acréscimo da pressão de preconsolidação do centro das entrelinhas em direção ao rodado e à projeção da copa das laranjeiras.

Os ensaios de compressão uniaxial são conduzidos a partir de amostras de solo com gradiente do conteúdo de água conhecido, por meio de secamento natural (Araujo-Junior, 2011; Souza et al., 2012) ou por meio de potenciais preestabelecidos (Debiasi et al., 2008; Araujo-Junior, 2011; Kamimura et al., 2012). Os dados de pressão de preconsolidação relacionados com o conteúdo de água no solo possibilitam descrever modelos de capacidade de suporte de carga para determinado manejo e camada de solo (Silva et al., 2002, 2006; Debiasi et al., 2008; Araujo-Junior, 2011; Souza et al., 2012). Uma vez que a densidade do solo (Ds) é obtida nessas mesmas amostras indeformadas, os modelos de capacidade de suporte de carga do solo podem ser descritos como função da Ds (Debiasi et al., 2008).

Até o momento não se dispõe de informações sobre a capacidade de suporte de carga de solos do arenito no noroeste do Paraná, que ocupam cerca de 3,2 milhões de hectares (Sá e Caviglione, 1999), dos quais aproximadamente $70 \%$ são ocupados por pastagens. As informações disponíveis sobre Ds, conteúdo de água e resistência do solo à penetração associados aos teores de carbono orgânico não são suficientes para caracterizar se determinado manejo conservacionista de solo nas entrelinhas dos pomares de laranja estariam promovendo maior capacidade de suporte de carga do solo com textura arenosa na camada superficial (Fidalski et al., 2010). 
Assim, o objetivo deste trabalho foi avaliar a capacidade de suporte de carga por meio da pressão de preconsolidação e sua dependência ao conteúdo de água, densidade e carbono orgânico de um Argissolo Vermelho distrófico latossólico, após 18 anos de implantação de plantas de cobertura permanentes nas entrelinhas de um pomar de laranja.

\section{MATERIAL E MÉTODOS}

O estudo foi desenvolvido em um experimento implantado em 1993, na Fazenda São Judas Tadeu, pelo Instituto Agronômico do Paraná (IAPAR), no município de Alto Paraná, região noroeste do Paraná $\left(23^{\circ} 05^{\prime}\right.$ sul; $52^{\circ} 26^{\prime}$ oeste; e $450 \mathrm{~m}$ de altitude). O clima na região é do tipo Cfa (Caviglione et al., 2000), com precipitações pluviais médias anuais de $1.400 \mathrm{~mm}$. O solo é um Argissolo Vermelho distrófico latossólico textura arenosa/média (Fidalski et al., 2010; Santos et al., 2013), com teores de argila nas camadas de 0,00-0,10 e 0,10-0,20 m, respectivamente, de 87 e $122 \mathrm{~g} \mathrm{~kg}^{-1}$.

A implantação do experimento se deu com o preparo do solo e a calagem em 1993 (aração e gradagem). No plantio das laranjeiras, foi utilizada a variedade Pera (Citrus sinensis) enxertada no porta-enxerto limoeiro variedade Cravo (Citrus limonia). Antes da implantação, a área era usada com pastagem braquiária (Urochloa humidicola). $\mathrm{O}$ delineamento experimental foi de blocos ao acaso, com três repetições. Cada parcela experimental foi composta por 15 plantas, dispostas em três linhas de plantas, no espaçamento de $7 \times 4 \mathrm{~m}$. Os tratamentos avaliados foram: gramínea - grama mato-grosso ou grama-batatais (Paspalum notatum); leguminosa - amendoim-forrageiro (Arachis pintoi); $\mathrm{e}$ vegetação espontânea - caracterizada pela vegetação espontânea manejada com herbicida pós-emergente entre 1996 e 2000 (Auler et al., 2008).

Anualmente, os três tratamentos foram roçados e os resíduos deixados sobre as entrelinhas do pomar, principalmente no final do período das águas (março). Nas linhas das plantas, o manejo foi realizado com herbicida pós-emergente em todos os tratamentos. A partir de 2008, foram realizadas roçadas das vegetações permanentes das entrelinhas do pomar de laranja e houve infestação progressiva de braquiária ( $U$. brizantha) nas entrelinhas do pomar. Para os tratos fitossanitários, calagem superficial, adubação, controle da vegetação e colheita de frutos, foram utilizados trator de pneu, pulverizador com capacidade de $2.000 \mathrm{~L}$ e roçadora mecânica, com massas aproximadas de 4.000, $1.000 \mathrm{e}$ $500 \mathrm{~kg}$, respectivamente. Estima-se que em 18 anos houve 250 tráfegos de máquinas (Tersi e Rosa, 1995) sobre os três tratamentos nesse pomar de laranja com diferentes conteúdos de água no solo.
As amostras de solo foram coletadas em 09/10/2011 nas entrelinhas do pomar, em três transectos perpendiculares aos troncos das laranjeiras de cada parcela experimental, nas posições de amostragem rodado e entrerrodado das entrelinhas e nas camadas de 0,00-0,10 e 0,10-0,20 m, totalizando 108 amostras indeformadas de solo. Essas amostras foram coletadas com trado tipo Uhland e cilindros volumétricos metálicos $(0,025 \mathrm{~m}$ de altura e 0,075 m de diâmetro), com umidade do solo próxima à capacidade de campo.

As amostras de solo foram saturadas em água e submetidas aos seguintes potenciais para a padronização do conteúdo de água do solo para os ensaios de compressão uniaxial: $-80 \mathrm{hPa}$ em mesa de tensão; e aos potenciais de -330 e -1.000 $\mathrm{hPa}$ em placas porosas em câmaras de Richards, utilizando-se aleatoriamente uma amostra de cada transecto, posição de amostragem e camada de solo para cada um dos potenciais. Após o equilíbrio em cada potencial, as amostras de solo foram submetidas a ensaios de compressão uniaxial em um consolidômetro digital (Silva et al., 2007), aplicando-se pressões subsequentes de 25, 50, 100, $200,400,800$ e $1.600 \mathrm{kPa}$ até obterem $90 \%$ da deformação máxima em cada pressão (Taylor, 1948), que se deu em um tempo de 5 min para cada uma dessas pressões, sem efetuar o descarregamento das pressões previamente aplicadas (Larson e Gupta, 1980), e obtida a deformação da amostra submetida a cada pressão. Após a liberação da pressão, as amostras foram secas em estufa a $105^{\circ} \mathrm{C}$ por $24 \mathrm{~h}$ para determinar a densidade do solo (Ds) conforme Claessen (1997). Em seguida, as amostras de solo foram tamisadas em peneira de $2 \mathrm{~mm}$ de diâmetro de malha para definir os teores de carbono orgânico (CO) do solo pelo método Walkley-Black (Claessen, 1997).

A pressão de preconsolidação $\left(\sigma_{p}\right)$ foi determinada após se obterem as curvas de compactação do solo, pela razão de vazios (e) em função do da pressão aplicada. Os ajustes foram realizados seguindo um modelo sigmoidal similar à equação de van Genuchten (van Genuchten, 1980), descrito por Baumgartl e Köck (2004) e detalhado em Cavalieri et al. (2008) para a curva de compactação do solo: $\{e=(e s-e r)[1+(\alpha \sigma) n]-(1-1 / n)+e s\}$, sendo $e$ a razão de vazios, $\sigma$ a pressão aplicada $(\mathrm{kPa})$ e $\alpha, e s$, er e $n$ os parâmetros de ajuste. Além disso, er $(0,27)$ foi usado como razão de vazios mínima ou residual (Baumgartl e Köck, 2004) quando o parâmetro não foi ajustado. Os coeficientes da curva sigmoidal foram considerados estatisticamente significativos pelo intervalo de confiança, em que o valor zero não foi incluído (Glantz e Slinker, 1990). A pressão de preconsolidação foi então obtida conforme Baumgartl e Köck (2004).

Os ajustes das equações para obter as pressões de preconsolidação foram realizados com o software SAS (SAS, 2000). Posteriormente, os 108 dados de 
pressão de preconsolidação, conteúdo de água, Ds e CO foram submetidos às análises de variâncias e testes de comparações de médias pelo modelo matemático de blocos casualizados no esquema de parcelas subdivididas (Banzatto e Kronka, 2006), alocando os três tratamentos nas parcelas e cada uma das três amostras dos transectos nas subparcelas, individualmente para cada camada e posição de amostragem de solo. Os conteúdos de água do solo dos três potenciais foram comparados pelo intervalo de confiança da média. Foram testadas as regressões da pressão de preconsolidação em razão do conteúdo de água, da Ds e do CO do solo. O grau de confiança utilizado para as análises estatísticas foi de $5 \%$.

\section{RESULTADOS E DISCUSSÃO}

A análise de variância indicou que não houve interação significativa entre os tratamentos e as três amostras de solo por parcela experimental para a Ds, o CO e a pressão de preconsolidação do solo $(p<0,05)$. A Ds não se diferenciou entre os tratamentos, as posições de amostragem e as camadas de solo (Quadro 1). Há relação direta da Ds com a pressão de preconsolidação em razão do manejo de solo, tendo sido verificada essa maior pressão na camada superficial em sistemas de manejo de plantas invasoras em lavoura cafeeira (Araujo-Junior et al., 2011) ou pelas operações realizadas com o trator (Kamimura et al., 2012); contrariamente, ambos podem diminuir com plantas de cobertura de inverno sem tráfego (Debiasi et al., 2008), Contudo, neste trabalho a pressão de preconsolidação foi dependente somente dos tratamentos.

$\mathrm{O}$ teor de $\mathrm{CO}$ do solo também não se diferenciou entre os tratamentos, as posições de amostragem e as camadas de solo (Quadro 1). A homogeneidade entre os valores de Ds e os teores de CO do solo para os três sistemas conservacionistas é atribuída à manutenção da gramínea, leguminosa e vegetação espontânea, manejadas por meio de roçadas sobre as entrelinhas do pomar de laranja sem nenhum revolvimento do solo (Auler et al., 2008).

$\mathrm{O}$ valor médio da pressão de preconsolidação foi maior no tratamento com gramínea $(185 \mathrm{kPa})$ em relação à leguminosa $(137 \mathrm{kPa})$ e vegetação espontânea $(139 \mathrm{kPa})$, sob o rodado na camada de 0,00-0,10 m (Quadro 1). Iori et al. (2014) também constataram maior pressão de peconsolidação nessa posição de amostragem de solo em lavoura cafeeira em Latossolo Vermelho-Amarelo com textura argilosa. Quanto à posição de amostragem, outros autores verificaram também maior pressão de preconsolidação na linha de tráfego do rodado, em lavouras de cana-de-açúcar (Kamimura et al., 2012) e café (Souza et al., 2012). Em relação à profundidade, Assis e Lanças (2005) também
Quadro 1. Densidade (Ds), carbono orgânico (CO) e pressão de preconsolidação $\left(\sigma_{p}\right)$ do solo amostrados em duas profundidades no local do rodado e do entrerrodado da máquina na entrelinha do pomar de laranja variedade Pera, com três tipos de cobertura vegetal na entrelinha

\begin{tabular}{|c|c|c|c|}
\hline Cobertura vegetal & Ds & $\mathrm{CO}^{(1)}$ & $\sigma_{p}$ \\
\hline & $\mathrm{kg} \mathrm{dm}^{-3}$ & $\mathrm{~g} \mathrm{dm}^{-3}$ & $\mathrm{kPa}$ \\
\hline & \multicolumn{3}{|c|}{ Rodado $(0,00-0,10 \mathrm{~m})$} \\
\hline Paspalum notatum & $1,66 \mathrm{a}$ & $5,13 \mathrm{a}$ & $185 \mathrm{a}$ \\
\hline Arachis pintoi & $1,66 \mathrm{a}$ & $4,00 \mathrm{a}$ & $137 \mathrm{~b}$ \\
\hline Vegetação espontânea & $1,67 \mathrm{a}$ & $5,17 \mathrm{a}$ & $139 \mathrm{~b}$ \\
\hline \multirow[t]{2}{*}{ CV (\%) } & 2,63 & 34,89 & 17 \\
\hline & \multicolumn{3}{|c|}{ Rodado $(0,10-0,20 \mathrm{~m})$} \\
\hline Paspalum notatum & $1,72 \mathrm{a}$ & $4,35 \mathrm{a}$ & $209 \mathrm{a}$ \\
\hline Arachis pintoi & $1,75 \mathrm{a}$ & $3,17 \mathrm{a}$ & $178 \mathrm{a}$ \\
\hline Vegetação espontânea & $1,74 \mathrm{a}$ & $3,04 \mathrm{a}$ & $196 \mathrm{a}$ \\
\hline \multirow[t]{2}{*}{ CV (\%) } & 2,16 & 46,42 & 25 \\
\hline & \multicolumn{3}{|c|}{ Entrerrodado $(0,00-0,10 \mathrm{~m})$} \\
\hline Paspalum notatum & $1,60 \mathrm{a}$ & $5,21 \mathrm{a}$ & $138 \mathrm{a}$ \\
\hline Arachis pintoi & $1,65 \mathrm{a}$ & $4,22 \mathrm{a}$ & $230 \mathrm{a}$ \\
\hline Vegetação espontânea & $1,64 \mathrm{a}$ & $3,86 \mathrm{a}$ & $220 \mathrm{a}$ \\
\hline \multirow[t]{2}{*}{ CV (\%) } & 4,79 & 32,16 & 79 \\
\hline & \multicolumn{3}{|c|}{ Entrerrodado $(0,10-0,20 \mathrm{~m})$} \\
\hline Paspalum notatum & $1,66 \mathrm{a}$ & $4,43 \mathrm{a}$ & $228 \mathrm{a}$ \\
\hline Arachis pintoi & $1,69 \mathrm{a}$ & $3,43 \mathrm{a}$ & $193 \mathrm{a}$ \\
\hline Vegetação espontânea & $1,66 \mathrm{a}$ & $3,26 \mathrm{a}$ & $170 \mathrm{a}$ \\
\hline CV (\%) & 3,33 & 41,58 & 30 \\
\hline
\end{tabular}

(1) CO: carbono orgânico pelo método Walkley-Black. Médias com a mesma letra não diferem entre si pelo teste Tukey $(p<0,05)$. CV: coeficiente de variação.

notaram maior capacidade de suporte na camada superficial de 0,00-0,05 $\mathrm{m}$ do que na de 0,10-0,15 m.

A ausência de diferença entre os tratamentos para a pressão de preconsolidação nas demais posições de amostragem e camadas de solo (Quadro 1) se deve aos elevados coeficientes de variação (CV) (25 a $79 \%)$, enquanto para o tratamento com gramínea sob o rodado na camada de $0,00-0,10 \mathrm{~m}$ o CV da pressão de preconsolidação foi de $17 \%$ (Quadro 1). A alta variabilidade da pressão de preconsolidação também foi relatada por Baumgartl e Köck (2004), em virtude da elevada heterogeneidade entre repetições.

Os valores das pressões de preconsolidação foram extremamente altos para a maioria dos tratamentos, das camadas e do rodado e do entrerrodado (Quadro 1), quando comparados às pressões de preconsolidação obtidas por Horn e Fleige (2003). Tal fato pode ser em razão das características do solo estudado, que possui classe textural arenosa nas camadas analisadas (teores de argila de 87 e $122 \mathrm{~g} \mathrm{~kg}^{-1}$, respectivamente nas camadas de $0,00-0,10$ e $0,10-0,20 \mathrm{~m}$ ), que geralmente 
são pouco compressíveis (Larson e Gupta, 1980). Os valores das pressões de preconsolidação sob o rodado e entrerrodado foram inferiores aos obtidos por Lima et al. (2004) em um pomar de laranja, respectivamente 298 e $309 \mathrm{kPa}$, após 10 anos de manejo com tráfego de trator de $3.900 \mathrm{~kg}$, em um Latossolo Vermelho-Amarelo distrófico com $170 \mathrm{~g} \mathrm{~kg}^{-1}$ de argila. Esses autores não verificaram diferenças estatísticas entre essas duas posições da amostragem na camada superficial de $0,075 \mathrm{~m}$.

Os conteúdos de água do solo apresentaram ampla variabilidade (Figura 1); contudo, não houve ajustes para descrever modelos de regressões para a capacidade de suporte de carga em função do conteúdo de água, tampouco em relação à Ds e o $\mathrm{CO}$ do solo ( $p<0,05$ e, ou, $\left.R^{2} \leq 0,20\right)$. A independência da pressão de preconsolidação ao conteúdo de água, à Ds e ao CO desse solo (Quadro 1) não possibilitou ajustes de modelos de capacidade de suporte desse solo arenoso, obtidos por outros autores em solos mais argilosos com teores de argila superiores a $256 \mathrm{~g} \mathrm{~kg}^{-1}$ (Silva et al., 2006; Debiasi et al., 2008; Araujo-Junior; 2011; Souza et al., 2012).

O tratamento com gramínea apresentou maior capacidade de suporte do solo sob o rodado, na camada de 0,00-0,10 m (Quadro 1). Isso indicou que os tratamentos com leguminosa e vegetação espontânea apresentam maior risco de compactação adicional, quando comparados à gramínea. Tais resultados reiteraram a importância do manejo conservacionista desses solos com gramíneas em pomares de laranja (Auler et al., 2008; Fidalski et al., 2010).

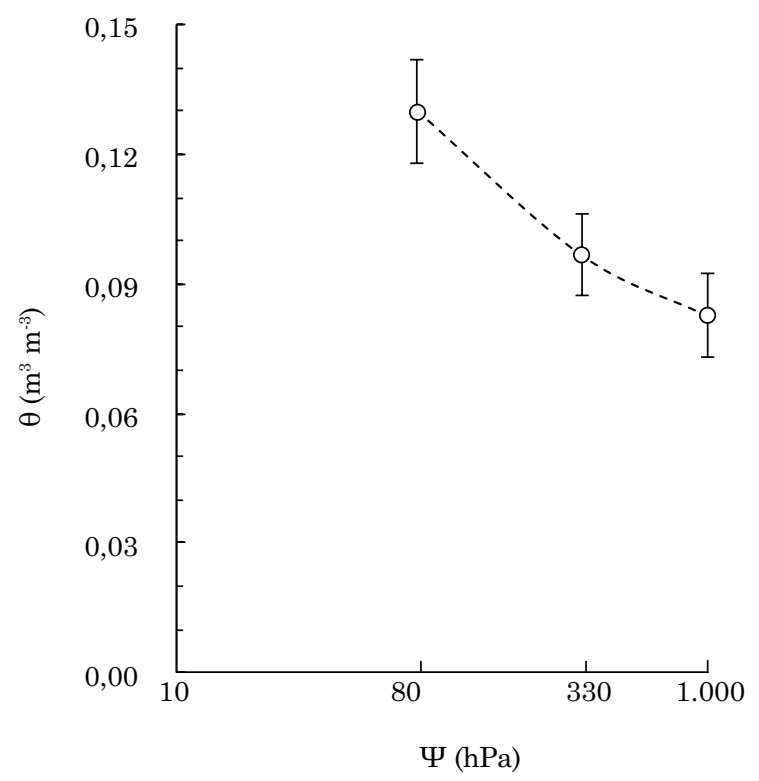

Figura 1. Conteúdo de água ( $(\theta)$ em três potencias ( $\Psi)$ das 108 amostras de solo utilizadas nos ensaios de compressão uniaxial. As barras verticais correspondem ao intervalo de confiança da média $(p<0,05)$.
Apesar de não ter ocorrido diferença significativa para pressão de preconsolidação entrerrodado na camada de 0,00-0,10 m (Quadro 1), os valores médios das pressões de preconsolidação para leguminosa e vegetação espontânea são considerados extremamente altos $(<150 \mathrm{kPa})$, enquanto para gramínea estariam dentro de uma faixa considerada muito alta (120-150 kPa), conforme os valores propostos por Horn e Fleige (2003). Isso sugeriu que compactações adicionais tenham já ocorrido para esses tratamentos, elevando sua capacidade de suporte de carga em áreas supostamente menos trafegadas, porém que sofreram os efeitos tridimensionais da transmissão de carga no solo (Gupta e Allmaras, 1987). Além disso, a similaridade nos valores médios de Ds e CO entre os tratamentos sugeriu que a morfologia de raízes e os seus efeitos sobre o sistema poroso do solo, decorrentes dos diferentes tratamentos, podem ter contribuído fortemente para a discrepância entre os valores médios de pressão de preconsolidação para gramínea, leguminosa e vegetação espontânea. Portanto, salienta-se a importância de estudos voltados a esses aspectos e sua influência na capacidade de suporte de carga do solo, principalmente em solos de textura arenosa, que são naturalmente menos estruturados que os de textura mais argilosa.

\section{CONCLUSÕES}

A pressão de preconsolidação em pomar de laranja não foi dependente do conteúdo de água, da densidade do solo e do teor de carbono orgânico do solo.

A pressão de preconsolidação em pomar de laranja sob o rodado na camada de 0,00-0,10 $\mathrm{m}$ foi menor com manejo por meio de roçadas nas entrelinhas com manutenção de leguminosa e vegetação espontânea do que com gramínea.

A manutenção permanente de gramínea manejada com roçadas nas entrelinhas do pomar de laranja proporcionou maior capacidade de suporte de carga do solo sob o rodado na camada superficial arenosa.

\section{AGRADECIMENTOS}

À Fundação Araucária pelas bolsas de Produtividade em Pesquisa e de Iniciação Científica concedidas, respectivamente, ao primeiro e quarto autores. Ao Conselho Nacional de Desenvolvimento Científico e Tecnológico (CNPq), pela bolsa de Produtividade em Pesquisa concedida ao segundo autor. À Fundação Araucária, pela bolsa de iniciação científica para Guilherme Anghinoni da 
Universidade Estadual de Maringá. À Pierina Inácio Ferrari e Filhos, pela cessão da área experimental na Fazenda São Judas Tadeu em Alto Paraná, PR.

\section{REFERÊNCIAS}

Araujo-Junior CF, Dias Junior MS, Guimarães PTG, Nunes Alcântara EN. Capacidade de suporte de carga e umidade crítica de um Latossolo induzida por diferentes manejos. R Bras Ci Solo. 2011;35:115-31.

Assis RL, Lanças KP. Avaliação da compressibilidade de um Nitossolo Vermelho distroférrico sob sistema plantio direto, preparo convencional e mata nativa. $\mathrm{R}$ Bras $\mathrm{Ci}$ Solo. 2005;29:507-14.

Auler APM, Fidalski J, Pavan MA, Neves CSVJ. Produção de laranja Pêra em sistemas de preparo do solo e manejo nas entrelinhas. R Bras Ci Solo. 2008;32:363-74.

Banzatto DA, Kronka SN. Experimentação agrícola. $4^{\text {a }}$.ed. Jaboticabal: FUNEP; 2006.

Baumgartl T, Köck B. Modeling volume change and mechanical properties with hydraulic models. Soil Sci Soc Am J. 2004;68:57-65.

Cavalieri KMV, Arvidsson J, Silva AP, Keller T. Determination of precompression stress from unixial compression tests. Soil Till Res. 2008;98:17-26.

Caviglione JH, Kiihl LRB, Caramori PH, Oliveira D. Cartas climáticas do Paraná. [CD-ROM]. Londrina: IAPAR; 2000.

Claessen MEE, organizador. Manual de métodos de análise de solo. $2^{\text {a }}$.ed. rev. atual. Rio de Janeiro: Embrapa Solos; 1997. (Documentos, 1).

Debiasi H, Levien R, Trein CR, Conte O, Mazurana M. Capacidade de suporte e compressibilidade de um Argissolo, influenciadas pelo tráfego e por plantas de cobertura de inverno. R Bras Ci Solo. 2008;32:2629-37.

Dias Junior MS. Compactação do solo. Tópicos Ci Solo. 2000;1:55-94

Fidalski J, Tormena CA, Silva ÁP. Least limiting water range and physical quality of soil under groundcover management systems in citrus. Sci Agric. 2010;67:448-53.

Glantz SA, Slinker BK. Primer of applied regression and analysis of variance. New York: McGraw-Hill; 1990.

Gupta SC, Allmaras RR. Models to access the susceptibility of soil to excessive compaction. Adv Soil Sci. 1987;6:65-100.

Horn R, Fleige H. A method for assessing the impact of load on mechanical stability and on physical properties of soils. Soil Till Res. 2003;73:89-99.

Imhoff S, Silva AP, Fallow D. Susceptibility to compaction, load support capacity, and soil compressibility of Hapludox. Soil Sci Soc Am J. 2004;68:17-24.
Iori P, Dias Junior MS, Ajayi AE, Guimarães PTG, Souza ZR, Figueiredo VC. Seasonal change of soil precompression stress in coffee plantation under sub-humid tropical condition. Coffee Sci. 2014;9:145-54.

Kamimura KM, Dias Júnior MS, Guimarães PTG, Santos GR, Oliveira MS. Capacidade de suporte de carga de um Latossolo Vermelho-Amarelo em uma lavoura cafeeira. R Bras Ci Solo. 2012;36:1457-65.

Larson WE, Gupta SC. Estimating critical stress in unsaturated soils from changes in pore water-pressure during confined compression. Soil Sci Soc Am J. 1980;44:1127-32.

Lima CLR, Silva AP, Imhoff S, Lima HV, Leão TP. Heterogeneidade da compactação de um Latossolo Vermelho-Amarelo sob pomar de laranja. R Bras Ci Solo. 2004;28:409-14.

Rucknagel J, Hofmann B, Paul R, Christen O, Hülsbergen KJ. Estimating precompression stress of structured soils on the basis of aggregate density and dry bulk density. Soil Till Res. 2007;92:213-20.

Sá JPG, Caviglione JH. Arenito Caiuá: Capacidade de lotação das pastagens. Londrina: IAPAR; 1999. (Informe da pesquisa, 132).

Santos HG, Jacomine PKT, Anjos LHC, Oliveira VA, Lumbreras JF, Coelho MR, Almeida JA, Cunha TJF, Oliveira JB. Sistema brasileiro de classificação de solos. $3^{\mathrm{a}}$.ed. Brasília: Embrapa Solos; 2013.

SAS Institute. SAS/GRAPH 2000 Software: Reference Version 8, v.2. Cary, NC: 2000.

Silva AR, Dias Junior MS, Guimarães PTG, Araújo Júnior CF. Modelagem da capacidade de suporte de carga e quantificação dos efeitos das operações mecanizadas em um Latossolo Amarelo cultivado com cafeeiros. R Bras Ci Solo. 2006;30:207-16.

Silva RB, Lanças KP, Masquetto BJ. Consolidômetro: equipamento pneumático-eletrônico para avaliação do estado de consolidação do solo. R Bras Ci Solo. 2007;31:607-15.

Silva VR, Reinert DJ, Reichert JM, Soares JM. Fatores controladores da compressibilidade de um Argissolo Vermelho-Amarelo distrófico arênico e de um Latossolo Vermelho distrófico. R Bras Ci Solo. 2002.26:1-8.

Souza GS, Souza ZM, Silva RB, Araújo FS, Barbosa RS. Compressibilidade do solo e sistema radicular da cana-de-açúcar em manejo com e sem controle de tráfego. Pesq Agropec Bras. 2012;47:603-12.

Taylor DW. Fundamentals of soil mechanics. New York: John Wiley \& Sons; 1948.

Tersi EA, Rosa SM. A subsolagem no manejo de solo para os pomares de citros. Laranja. 1995;16:289-98.

van Genuchten MT. A closed-form equation for predicting the hydraulic conductivity of unsaturated soils. Soil Sci Soc Am J. 44:892-8, 1980 .

Veenhof DW, Mcbride RA. Overconsolidation in agricultural soils. I. Compression and consolidation behavior of remolded and structured soils. Soil Sci Soc Am J. 1996;60:362-73. 